\title{
The rhythms of scholarly publication: suggestions for academic research administrators making bibliometric comparisons across disciplines
}

Anthony J. Olejniczak

Director, Academic Analytics Research Center (AARC)

https://orcid.org/0000-0002-4666-8933

William E. Savage

Senior Researcher, Academic Analytics Research Center (AARC)

https://orcid.org/0000-0002-7711-591X

Richard Wheeler

Graduate Dean Emeritus, University of Illinois at Urbana-Champaign

Senior Advisor, Academic Analytics, LLC

https://orcid.org/0000-0002-5388-4714

\section{A case for nuanced bibliometric data}

The need for research universities to understand expectations for scholarly publishing across a wide array of disciplines is vital to an institution's self-understanding and to decisionmaking arising from that understanding. Administrators, staff, and faculty members serve on college-level executive committees, provost-level promotion and tenure committees, and committees that oversee campus-wide recognition of research excellence. They are engaged in processes that allocate research funding in response to competing proposals from several fields, or that authorize and oversee the development of cross-disciplinary programs. They develop strategic plans that are shaped by an awareness the strengths, needs, opportunities, and 
vulnerabilities of units across the entire university. In all these processes, they are called upon to assess the scholarly output of faculty in disciplines that represent a wide variety of publication cultures. They see the vitas of faculty in many fields and in different ranks, and come to realize, for example, how different the $\mathrm{CV}$ of a chemist is from the $\mathrm{CV}$ of a historian. Over time experienced administrators and faculty develop an intuitive understanding of these differences. But even that understanding is shaped by a limited sample, without a national context.

Disciplinary differences in published outputs are not limited to disparate subject matter across schools and departments. The pace of publication and the preferred mode of dissemination vary widely across fields, further complicating the task of university administrators and faculty charged with comparison and assessment across disciplines. Peer-reviewed journal articles are commonly produced by nearly every research-focused academic program, but conference proceedings, books, chapters in edited volumes, and other modes of communication are also important to varying degrees in many disciplines, sometimes as the primary means of disseminating research. For example, the single-author article output of a humanist will be much smaller than that of a comparably well-published physical scientist with multiple co-authors. The pace of publication also varies - sometimes substantially - within the same broad area. In engineering, for example, data from Academic Analytics (AAD2019-1740) reveal that over the most recent ten-year period the median number of articles published by mechanical engineers at Ph.D. granting universities in the United States is 22 (2.2 article authorships per person per year), while their materials science and engineering colleagues published nearly twice as many articles over the same timeframe (a median of 40 articles, or 4.0 article authorships per person per year; data for all disciplines are available at https://osf.io/myaut/. Experienced committee members and administrators may be sensitive to these (sometimes subtle) disciplinary differences, but we 
believe this intuitive understanding is strengthened by data that reflect essential dimensions of discipline-based publication patterns across fields, each with its own distinctive publication culture. For bibliometric-based comparisons across fields to be useful, we suggest that data should represent accurately the differences among fields, be wider in scope than only journal articles, and incorporate discipline-specific rhythms of publication.

In the tables presented here (https://osf.io/myaut/), we show data pertaining to publication rates and publication venues across 170 academic disciplines. We model the publication patterns of faculty members at U.S. research universities at different career stages, and in so doing we hope to provide a nuanced and up-to-date reference for faculty members, administrators, and staff charged with interpreting publication outputs across disciplines and in a comparative context. Not all active scholars in any field will follow the same pattern of publication, and not all articles or books are of equal value: these data do not reflect quality measures. Our goal is not to establish a template against which individual scholars should be measured, but to contextualize a general picture of publication patterns representative of each discipline, with the knowledge that there will be notable individual variations.

Bibliometric data are not a replacement for human judgment, and scholarly publications represent only one group of tiles in the greater mosaic of a faculty member's activity. The data we share are not intended to devalue teaching, service, and other contributions to one's community and students. Our goal in making public the associated tables is not to increase institutional reliance on pertinent data, but (1) to demonstrate the importance of recognizing the distinctive publication practices of different disciplines, (2) to provide a fuller picture of how a range of publication venues differentially reflects scholarly dissemination in each discipline, and (3) to provide reliable ways to incorporate that recognition into institutional awareness. In short, 
we offer these data to give a fuller picture of the bibliometric component within the entire scope of faculty contributions and scholarly activity.

We gathered the discipline affiliations, professorial ranks, and publication histories of 198,989 scholars at 392 Ph.D. granting institutions in the United States from the Academic Analytics commercial database (database version AAD2019-1740). Published works are categorized into disciplines based on each scholar's department affiliation(s), rather than an $a$ priori subject classification of journal titles. For example, a faculty member whose research program focuses on developing new quantitative analytical methods may publish mainly in journals classified as "statistics" by such a priori schemes, while this person's academic appointment is in a psychology department. Classifying this scholar's discipline as "statistics" based on the venue of publication fails to capture the author's role as a professor within the psychology department - a role that is critical to the proper contextualization of the department's research.

\section{There's more than just journal articles}

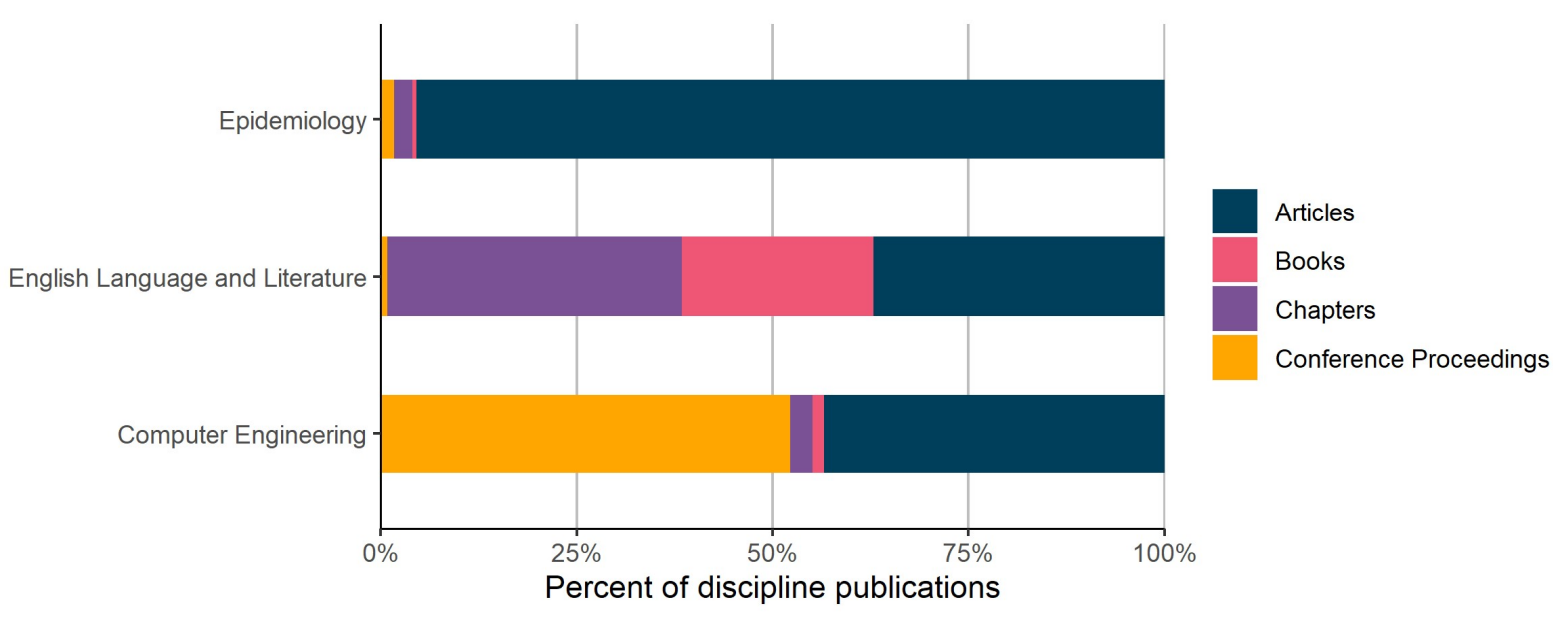

The proportion of each type of publication varies widely across disciplines. Three extreme examples are given in the figure above (based on ten years of publication data): Academic Analytics Research Center (AARC) 
epidemiologists publish mainly journal articles; more than $60 \%$ of English language and literature publications are books and book chapters in edited volumes; conference proceedings are published more often than any other mode of dissemination among computer engineers. A dataset containing the percentage breakdown for each discipline is available as Table 1 (https://osf.io/myaut/). Unfortunately, journal articles (and citations to journal articles) are the only type of publication accounted for in many comparative displays of research production. This is particularly true among widely consulted (if often maligned) university ranking schemes, effectively penalizing universities whose faculty disproportionately practice in disciplines that favor other modes of knowledge dissemination. By examining only journal articles, one fails to capture more than $50 \%$ of the published works in 26 of the 170 disciplines in our data, almost all of which are in the humanities. A singular focus on journal articles may also yield an incomplete view of bibliometric patterns in STEM disciplines like Computer Science and Electrical Engineering, where conference proceedings are more common than journal articles.

\section{How much do other scholars in my field publish?}

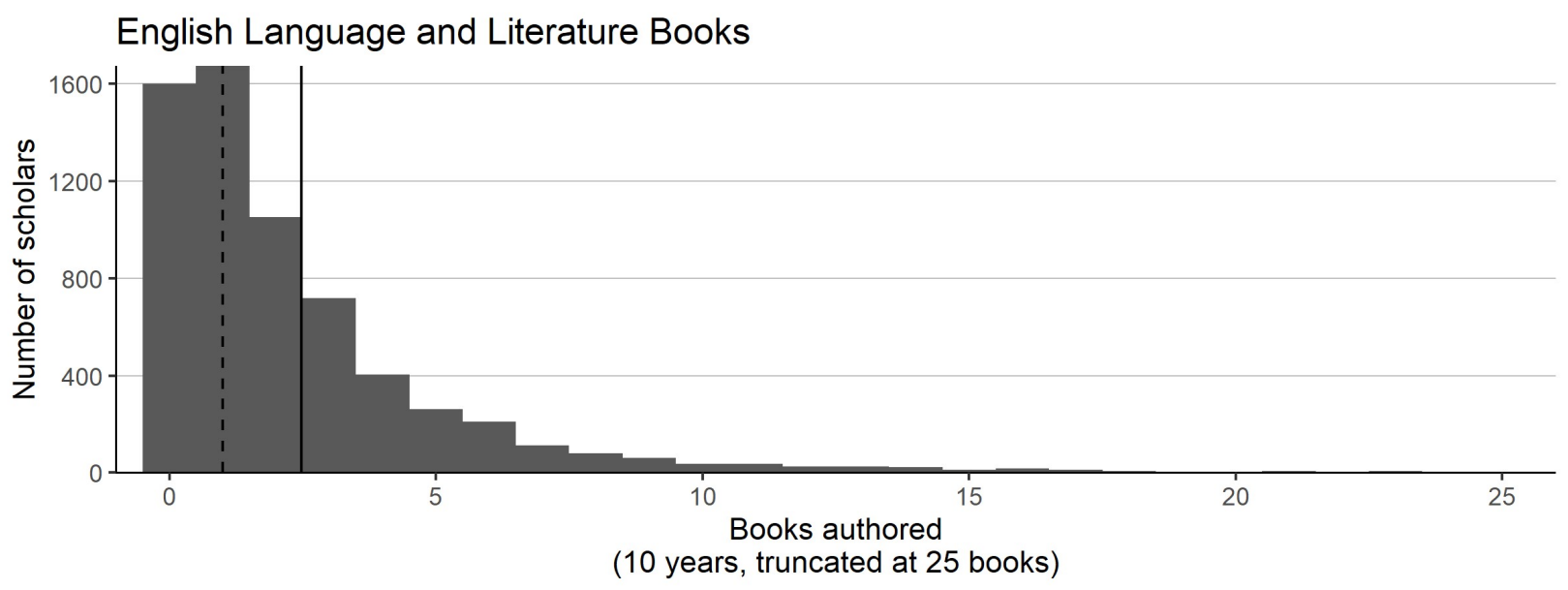

Another often overlooked characteristic of bibliometric data is skewed publication count distributions, evincing a long tail due to a small number of researchers publishing an Academic Analytics Research Center (AARC) 
exceptionally large volume of work. The plots above and below show this phenomenon for books in English Language and Literature and for journal articles in Physics. In each case, the solid vertical line representing the mean number of publications over 10 years is more than twice the median value, represented by the dotted vertical line. Evaluating publishing productivity based on discipline means may contribute to unrealistic comparisons and expectations, unduly influenced by a minority of prolific scholars. We suggest the median as a more appropriate measure of central tendency within a discipline, and we further recommend visualizing bibliometric data to aid the identification and interpretation of patterns. Table 2 shows the mean and median values for all four publication types over 10-year, 5-year, and 3-year timeframes, in all 170 disciplines (https://osf.io/myaut/).

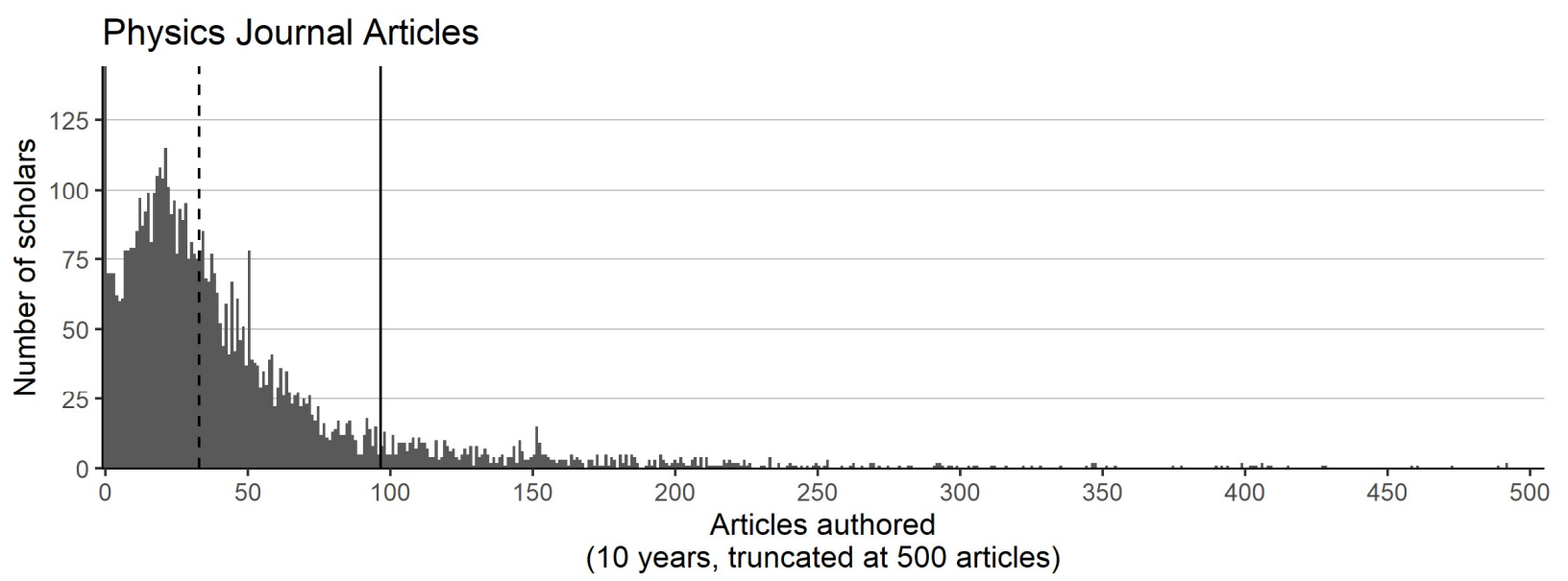

\section{The rhythm of publication}

Each discipline has a unique rate, or "rhythm" of publication. These discipline-specific publication rhythms reveal an important and, we believe, often overlooked aspect of bibliometric comparisons: one's choice of a comparative timeframe has a profound effect on comparisons across disciplines. In Table 3, for instance, we calculate that $81 \%$ of historians have published at least one book in the most recent 10-year period (https://osf.io/myaut/). Looking at only the most Academic Analytics Research Center (AARC) Page 6 of 10 
recent 5-year period, the percent of historians with a book publication drops to $56 \%$, and over the most recent three years only $42 \%$ of History faculty have authored a book. For most humanities disciplines, the median number of books per person published in a five-year period is zero, reflecting their long gestation time. Short timeframes fail to capture the rhythm of book publication among humanist scholars.

By comparison, the rhythm of conference proceeding publications in Computer Science is faster: $93 \%$ of computer scientists have at least one conference proceeding over 10 years, $89 \%$ over five years, and $86 \%$ over three years. Clearly, shorter timeframes have the potential to disadvantage some disciplines in a comparative context, particularly the humanities and other fields where books are a more common mode of knowledge dissemination. Interestingly, when analyses are limited to only journal articles, shorter timeframes still appear to disadvantage humanities disciplines, where articles are typically single-authored and relatively long. In German Language and Literature, $88 \%$ of scholars have published a journal article over a 10 year period, 79\% have published an article over a 5-year period, and 67\% have published an article over a 3-year period, a 21\% drop in the number of scholars whose work is captured when the timeframe is reduced from ten to three years. In Economics, however, the decline over the same timeframe is $7 \%$. We encourage all who are charged with using bibliometric data in an evaluative context to consider carefully which timeframes are appropriate to create a more accurate representation of scholarly publishing across disciplines.

\section{Professorial rank and publishing productivity}

Academic units are invariably composed of scholars at different career stages: senior, mid-career, and early career scholars. Publication productivity differs across these age cohorts, 
as access to resources and myriad other factors change throughout the course of one's career. We separated our data by professorial rank (Assistant, Associate, and Full Professor) to explore whether career stage impacts publishing trends; these data are presented in Table 4, wherein substantial inter-rank variation is observed (https://osf.io/myaut/). For example, the median journal article count over five years for an assistant professor of evolutionary biology is 14 articles, while the median count for a full professor in the same field is 25 articles $-\mathrm{a} 78 \%$ increase (this holds true even when considering only those assistant professors who earned their terminal degree five or more years ago). We described elsewhere how one's career stage is related to publication medium (DOI: 10.1007/s11192-021-03957-4), concluding that senior scholars are more likely to publish books and chapters in edited volumes than their younger colleagues in most fields. The data in Table 4 confirm this pattern, and indicate that even within a publication type, there are substantial differences in productivity between faculty at different career stages. The different career stages of the individual scholars comprising an academic unit should be considered when exploring the bibliometric outputs of those units individually in evaluations across disciplines.

\section{What becomes a bibliometric artifact?}

We demonstrated above that journal articles alone are inadequate to characterize publication productivity in many disciplines, but there are other types of omission one should consider when evaluating publications. In particular, scholarly works that are not registered as a bibliometric artifact - a persistent digital record with a unique identifier and other pertinent metadata such as a DOI or ISBN - are unlikely to be indexed by the bibliometric databases often used in university communities. It may also be the case that a discipline does not have a tradition 
of creating digital bibliometric artifacts for some classes of work. Data in Table 1, for instance, indicate that humanists do not author many publications recorded as conference proceedings. Humanists at research universities regularly attend and submit their work to conferences, presenting that work in papers they read or circulate to other attendees. In the humanities, however, conference presentations much less frequently are published as conference proceedings, and therefore do not become bibliometric artifacts as often as they do in other fields. For bibliometric comparisons, we need to move beyond asking "what journals does this database contain?" to the more nuanced question "what publication types are pertinent to a discipline, and of those, which become quantifiable artifacts?"

\section{Conclusions}

Bibliometric indicators are widely used at American research universities. In light of the extensive institutional use of such data, we hope these tables encourage those charged with bibliometric evaluations to frame more refined questions, taking into account disciplinary publication rhythms, preferred modalities of knowledge dissemination, appropriate comparative metrics, and the sometimes subtle differences in publication productivity across career stages. Bibliometric indicators are an important component in the context of the many other service and instructional responsibilities regularly assumed by faculty members at research universities across the country, and it is important that these indicators be understood with reference to the characteristic publication cultures of the different disciplines. 
Conflict of Interest Declaration: AJO and WES are paid employees of Academic Analytics, LLC, and RW is a paid consultant to Academic Analytics, LLC. None of the authors have an equity interest in Academic Analytics, LLC. The results presented reflect the authors' opinions, and do not necessarily reflect the opinions or positions of Academic Analytics, LLC. Academic Analytics, LLC management had no oversight or involvement in the project and were not involved in preparation or review of the manuscript. All work was done as part of the respective authors' research, with no additional or external funding. 